\title{
Innovative nanocrystal-based technologies for ceramic devices with novel electronic functions
}

\author{
Makoto KUWABARA* \\ 4-36-501 Shimoshirouzu-kita, Kasuga, Fukuoka 816-0854, Japan \\ Received July 1, 2012; Accepted July 5, 2012 \\ (C) The Author(s) 2012. This article is published with open access at Springerlink.com
}

\begin{abstract}
There has been a great progress in the synthesis of a variety of inorganic colloidal nanocrystals (NCs) with highly controlled size and shape in the last decade. This achievement has promoted comprehensive researches on the fabrication and engineering of $\mathrm{NC}$ devices with novel structure and function with the aim of utilizing them in a wide area of applications, including electronics and optoelectronics, by using various NC assembling and patterning methods. However, colloidal NCs having been extensively investigated so far are mostly metals, semiconductors and magnetic materials, and only limited investigations on oxide NCs from a point of view of NC ceramic engineering have been done. Here, innovative nanocrystal-based technologies necessary for the fabrication of $\mathrm{NC}$ ceramic devices with novel and gigantic electronic functions, limiting the materials to perovskite oxides, and a new perspective on the future ceramic technology are discussed.
\end{abstract}

Key words: nanocrystals; electronic functions; Perovskite; bottom-up process

\section{Introduction}

There has been a great development in the ceramic technology based on powder processing in the last three decades, and ceramic devices with a variety of electronic functions have been manufactured thereby. The powder-processing-based ceramic technology owes its striking development to marked progresses in both technologies of producing starting ceramic powder and of forming device structures. The early ceramic powder synthesis technology, based on solid-state reaction methods, has made a rapid advance by adopting the advantage of solution-phase synthesis

* Corresponding author.

E-mail: m-n.kuwabara@song.ocn.ne.jp methods, which allow production of ceramic powder with high purity and much homogeneous grain morphologies (size and shape). It is now possible to obtain various kinds of electronic ceramic powder with well-controlled particle sizes in the range from nanometer to micrometer by employing suitable solution methods, and one may easily find many reports on the synthesis of nanocrystalline particles of perovskite oxides, which are used in the largest quantity in the electroceramic industry [1-4]. As to the technology of fabricating device structures, since most electronic ceramic devices have been used in rather simple forms of disks and slabs unless their forms cannot give desired electronic functions, the development of techniques for making two- and three-dimensional functional structures with purpose of generating new electronic functions has little 
progressed. However, from a keen demand for continuing downsizing of ceramic devices, such as capacitors and inductors, used in large quantity as key elements in microelectronic circuits, the technology of forming and stacking thin ceramic (green) sheets into a multilayer structure with a uniform thickness of sintered ceramic layers (namely, interelectrode spacing) has greatly advanced to allow successful production of multilayer ceramic chip devices with highly enhanced electronic functions. The state-of-the-art technology enables to fabricate a multilayer structure with an interelectrode spacing of around $1 \mu \mathrm{m}$ [5], and the commercialization of multilayer ceramic chip capacitors, with the smallest size of $2012(2.0 \mathrm{~mm} \times$ $1.25 \mathrm{~mm}$ ), with extremely large capacitance values of $>22 \mu \mathrm{F}$ has recently been realized by Murata Co. Similar fabrication techniques for multilayer tip-type devices have been applied to other electronic ceramics, including thermistors [6], varistors [7], and inductors [8], resulting in a considerable success in the improvement of the performance of their devices. However, since the current powder processing-based ceramic technology has substantial technical limitations that hardly allow creating two- and threedimensional ceramic nanostructures with feature sizes of $<100 \mathrm{~nm}$, it is essential to develop innovative nanocrystal-based ceramic technology comparable with the photolithography-based semiconductor (or silicon) technology to open a new horizon in the field of electronic ceramics.

Photolithography as the central technique in the semiconductor technology has been most successful in manufacturing microelectronic structures, by which it is technologically possible to fabricate almost all three-dimensional even complex microstructures designed to let new phenomena occur. However, little benefit can be derived from the semiconductor technology when used to fabricate ceramic nanostructures because of great disadvantages with its time-consuming and costly processing and poor applicability to ceramic materials of particularly multicomponent systems. For further developments in the electronic ceramic industry, an alternative technology of manufacturing ceramic nanostructures needs to be developed, but such technology has not systematically and intensively been investigated yet.

To resolve this issue it would be most effective to establish a robust technology of assembling NCs into electronic ceramic devices with novel functional structures. In this regard, there have been extensive researches on the methods for organizing (assembling) NCs into specific ordered arrays and patterned structures toward various electronic applications. By various $\mathrm{NC}$ assembling processes with and without combination of lithography-assisted patterning methods novel three-dimensional $\mathrm{NC}$ structures, including $\mathrm{NC}$ superlattices as a typical example, and some electronic and optoelectronic NC devices have been successfully fabricated. Detailed and comprehensive information of these NC superlattices and devices can be found in some articles [9-12].

Here, we study a subject on the fabrication of $\mathrm{NC}$ ceramic devices with gigantic electronic functions (GEFs), including relaxor and PCTR (positive temperature coefficient of resistivity), derived from novel nanostructures constructed, taking such a nanostructure, as a model system, as is schematically shown in Fig. 1, and discuss sophisticated techniques required to fabricate such $\mathrm{NC}$ devices, limiting the materials of NCs to perovskite oxides, and technical problems to be solved.

\section{Synthesis of Perovskite NCs with a cubic shape (nanocubes)}

There have been numerous researches on the synthesis of colloidal monodisperse NCs with well-controlled morphology and structure of a wide variety of materials, including metals $[13,14]$, semiconductors $[14,15]$, and oxides $[14,16]$, using various solutionphase methods such as hydrothermal/solvothermal [17],

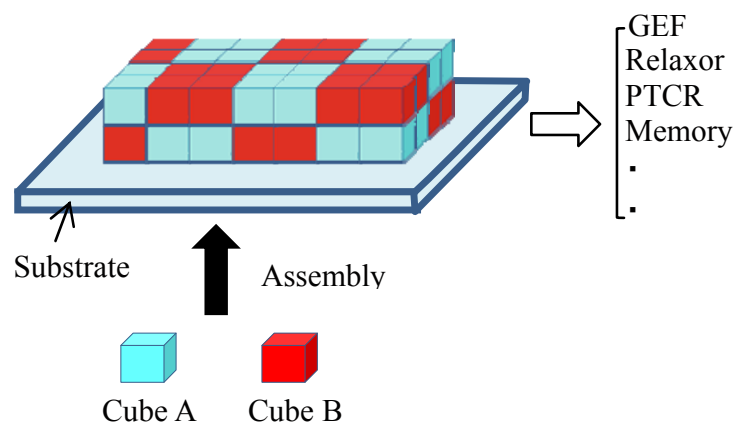

Fig. 1 A schematic image of a NC device (nanostructure) fabricated using $\mathrm{NC}$ cubes and examples of gigantic electronic functions (GEFs) derived from novel nanostructures. Assembly of nanocubes is assumed to be carried out using those monodispersed in proper solutions. PTCR denotes Positive Temperature Coefficient of Resistivity. 
hot-injection/decomposition [18], sol-gel (aqueous/ nonaqueous) [19], and liquid-solid-solution [20] routes. To fabricate NC devices with novel structure and function it is first of all necessary to produce NCs with precisely controlled morphology and structure, and the size and shape control of NCs has been achieved to a large extent for metal, magnetic and semiconductor materials. Murray group has succeeded in the synthesis of a variety of colloidal nanoparticles with precise values of size and shapes designed, and they have accomplished most impressive achievements in the fabrication of three-dimensional binary nanoperticle (nanocrystal) superlattices (BNSLs) with diverse structures, using spherical nanoparticles with different sizes [9]. In Fig. 2 are shown some examples of BNSLs fabricated by his group using a self-assembly process based on the solvent-evaporation-deposition (SED) method mentioned below, in which their TEM images of the characteristic projections and modeled unit cells of the corresponding 3-D structures are presented.

In previous researches on the fabrication of functional nanostructures spherical nanoparticles have mainly been used; however, to create highly dense NC structures cubic NCs (NC cubes) are favorable for using as building blocks since assembly of $\mathrm{NC}$ cubes theoretically allow creation of a NC structure of nearly full dense, as seen from the schematic shown in Fig. 1.
As to the synthesis of perovskite oxide nanoparticles, including $\mathrm{BaTiO}_{3}$ and $\mathrm{SrTiO}_{3} \mathrm{NCs}$, there have appeared a large number of reports in the literature; however, limiting the items to monodisperse $\mathrm{BaTiO}_{3}$ and $\mathrm{SrTiO}_{3}$ nanocubes, related reports are quite small in number. Examples of monodisperse $\mathrm{BaTiO}_{3}$ and

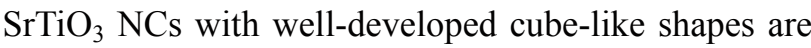
shown in Fig. 3; the $\mathrm{BaTiO}_{3}$ nanocubes were synthesized by a soft solution chemical process using $\mathrm{Ba}\left(\mathrm{NO}_{3}\right)_{2}, \mathrm{Ti}(\mathrm{OBu})_{4}, \mathrm{BuOH}$ and oleic acid at $135^{\circ} \mathrm{C}$ under a strong $\mathrm{NaOH}$ basic condition [21], while the $\mathrm{SrTiO}_{3}$ nanocubes were prepared from an aqueous solution containing $\mathrm{Sr}(\mathrm{OH})_{2}$, bis(ammonium lactate) titanium dihydroxide (TALH), hydrazine and oleic acid using a hydrothermal process at $200{ }^{\circ} \mathrm{C}$ under a strong $\mathrm{NaOH}$ basic condition [22]; the latter was modified from that proposed by Koumoto group [23]. It can be presumed that organic molecules used in the solvothermal/hydrothermal processes, which act as ligand and/or surfactant molecules, play important roles in the formation of cubic NCs in these cases, but the forming mechanisms of these NC cubes have not been satisfactorily clarified yet. A strict understanding of the NC size/shape-control mechanism in these rather simple systems, by carrying out much closer investigations under strictly controlled synthesis conditions, will lead to a significant success in the synthesis of high quality monodisperse perovskite
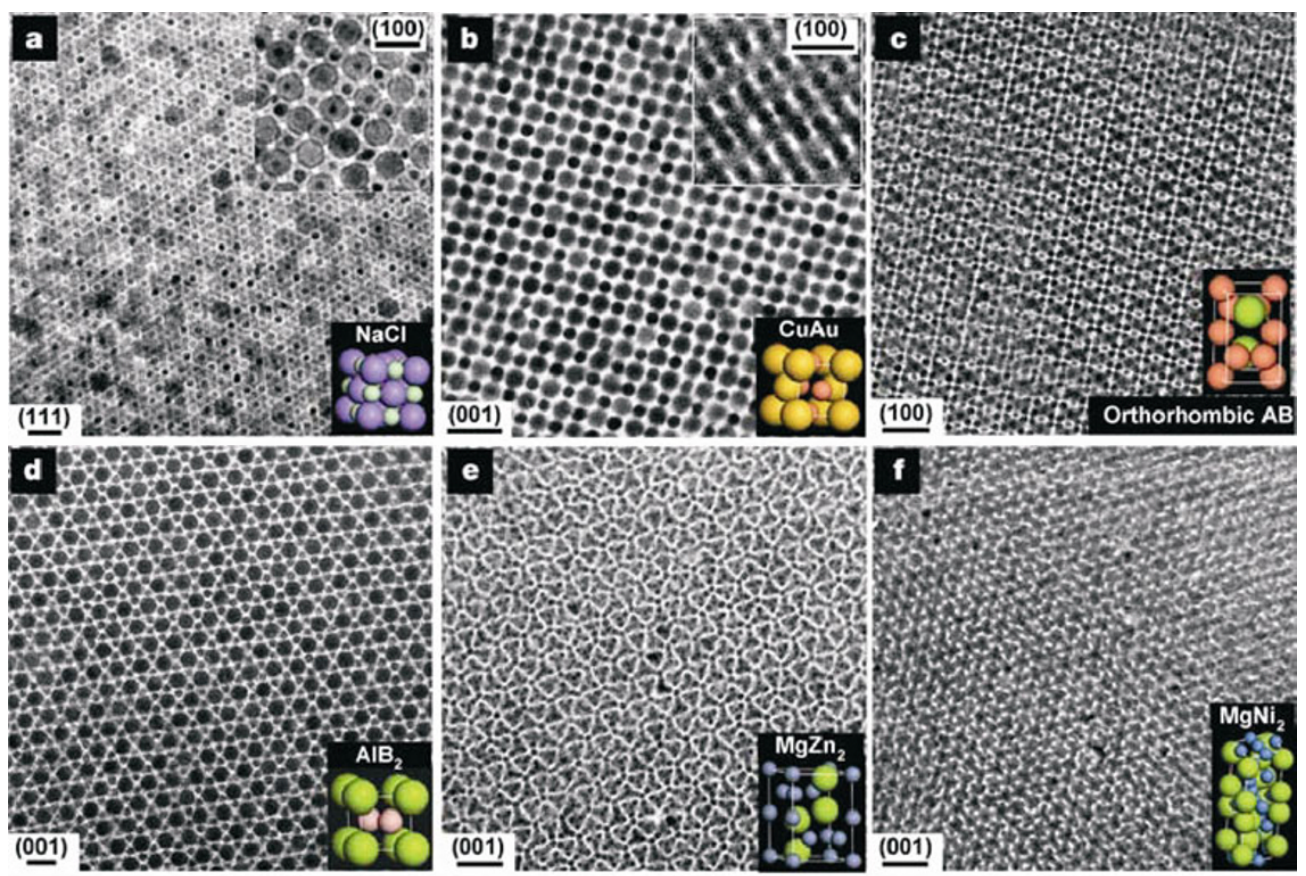

Fig. 2 TEM images of the characteristic projections of binary superlattices (BNSLs), self-assembled from different NCs, and modeled unit cells of the corresponding 3-D structures. Reprinted from Ref. [9]. 


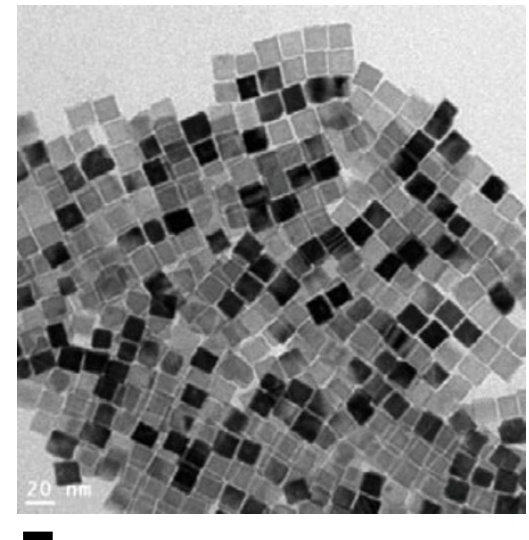

(a)

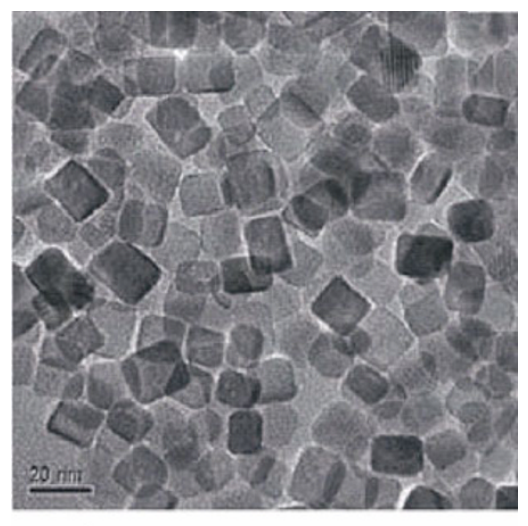

(b)

Fig. 3 TEM images of (a) $\mathrm{BaTiO}_{3}$ nanocubes, reprinted from Ref. [21] and (b) $\mathrm{SrTiO}_{3}$ nanocubes., reprinted from Ref. [22]. Bars $=20 \mathrm{~nm}$.

nanocubes with a size desired and its narrow size distribution.

\section{Assembly of NC cubes into functional nanostructures}

There have been a variety of methods for fabrication of $\mathrm{NC}$ devices with electronic functional structures using various NC assembly techniques such as SED [24], liquid-air-interface (LAI or LB: Langmuir-Blodgett) [25], spin-coating/dip- coating layer-by-layer (LBL) [26], and surfactant-mediated-aggregation (SMA) [27] processes, together with lithography-assisted patterning processes. Of these, outlines of SED, LAI and SMA processes are schematically shown in Fig. 4. Since the methods of spin-coating and dip-coating processes are simple and unnecessary to be described, schematics of these processes are not shown in the figure. As mentioned above, it has been proved that the SED-based assembly process enables to construct excellent BNSL structures. This method is simple and easy to use for assembling particularly spherical NCs into dense (nearly close-packed) nanostructures, but that it requires a strict control of the evaporation condition of solvent to fabricate free-of-disorders BNSLs should also be mentioned. Dong et al. [25] has successfully fabricated BNSL magnetoresistive devices using a LAI technique; in Fig. 5 are shown schematics of the processes of BNSL membrane growth and transferring it on a substrate prepatterned with metal electrodes in (a) and the device fabricated in (b). The LAI method can be used for NCs with various shapes such as spheres, cubes, and rods, and has its advantages of allowing the construction of large-area single- and multi-component $\mathrm{NC}$ membranes on various kinds of substrate including thin polymer sheets; the membrane growth and transfer processes
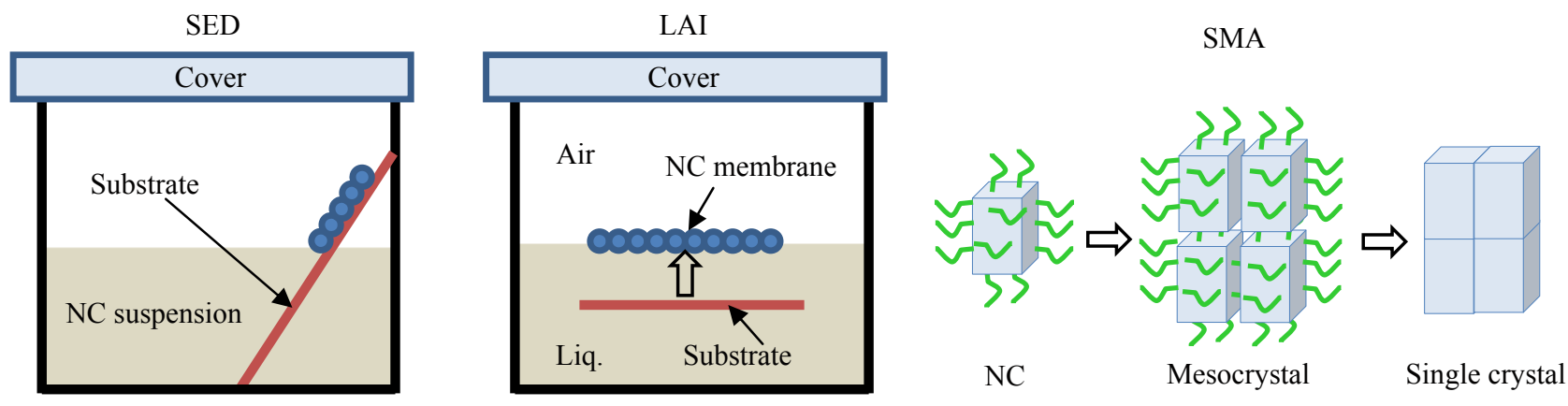

Fig. 4. Schematics of solvent-evaporation-deposition (SED), liquid-air-interface (LAI) and surfactant-mediated-aggregation (SMA) processes. The SMA process describes a growth mechanism of single crystals, in which a mesocrystal intermediate is formed by surfactant-mediated-assembly of NCs, then transforming into a single crystal by crystallographic fusion of the oriented building units through the oriented attachment mechanism. 


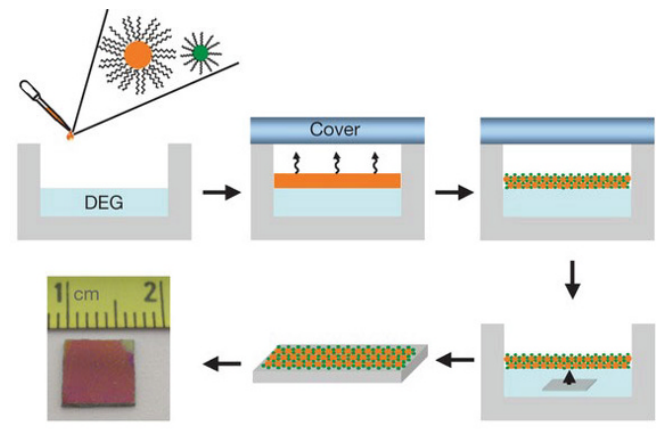

(a)

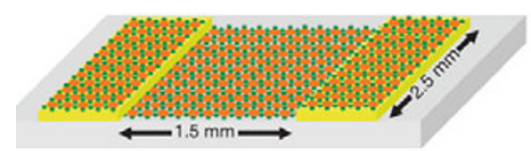

(b)

Fig. 5 (a) Schematics of a BNSL membrane growth and transfer process onto a substrate and (b) a NC device fabricated. Reprinted from Ref. [25].

need to be properly modified according to the system of NCs used, though. The LAI method may significantly enhance its advantage by involving role-to-role processing. The LBL method combined with spin-coating and dip-coating processes has also been widely used for fabricating $\mathrm{NC}$ devices, and many examples of successful fabrication of electronic/optoelectronic NC devices, such as field-effect transistors, photodetectors, and solar cells, have appeared in the literature $[28,29]$. However, since spin-coating and dip-coating methods are considered to hardly allow assembling of NC cubes into nearly full dense membranes, these methods are not suitable for the fabrication of ceramic devices using nanocubes. As to the SMA process, it describes a growth mechanism of single crystals via a mesocrystal intermediate formed by surfactant-mediated-assembly of NCs, which then transform into a single crystal by crystallographic fusion of the oriented building units through the oriented attachment (OA) mechanism, as shown in Fig. 4c. It may be worthy to note that mesocrystals have characteristic facetted external structures, and a nearly perfect crystallographic alignment of the constituent NCs can be seen in them. The growth mode of single crystals by the OA mechanism, which is different from a classical one known as the Ostwald ripening mechanism, was first reported by Banfield et al. [30] for the case of anatase $\left(\mathrm{TiO}_{2}\right)$ aggregates composed of coarsened primary nanoparticles obtained under hydrothermal aging conditions. Since then, many reports on the OA phenomenon observed for $\mathrm{NC}$ aggregates of a variety of materials, including $\mathrm{CeO}$ and $\mathrm{ZnO}$, synthesized via solution-phase routes have appeared in the literature [31,32]. The SMA process associated with the phenomenon of OA-based crystallographic fusion between NC particles is expected to allow a successful fabrication of $\mathrm{NC}$ structures with high electronic transport characteristics, leading to the development of intriguing and sophisticated techniques for fabricating $\mathrm{NC}$ ceramic devices with superior electronic functions. One may found comprehensive and much detailed descriptions of SMA and OA in some articles and reviews $[33,34]$.

Figure 6 shows a schematic of a route that the author propose to the fabrication of $\mathrm{NC}$ ceramic devices with novel electronic function-bearing structures on polymer sheets via an LAI or SMA method, in which charged NC cubes to be assembled and transferred on a substrate by electrostatic forces are used as building blocks. There have been many investigations on the creation of $\mathrm{NC}$ structures using

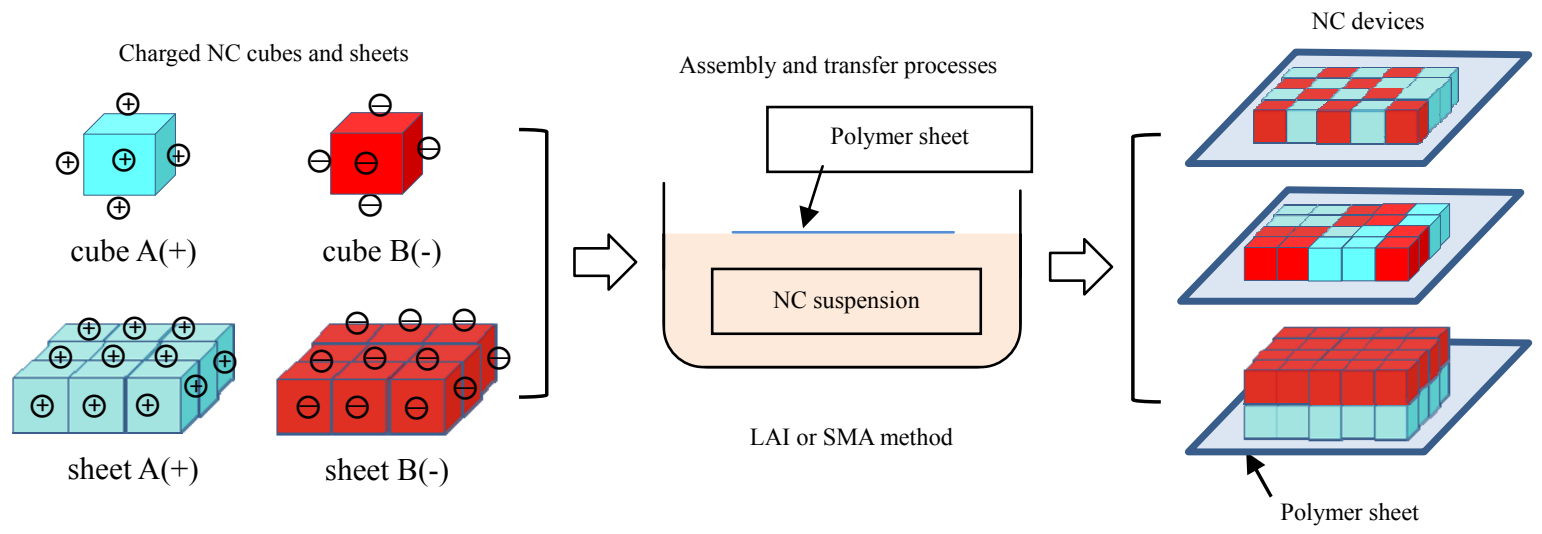

Fig. 6 Schematic of a method for the construction of NC devices on polymer sheets, in which charged NC cubes and electrostatic-force-based assembly and transfer processes by the LAI or SMA method are used. 
electrostatic forces, and successful fabrication of many unique nanostructures, including binary superstructures, has been reported $[35,36]$. However, since the shape of charged nanoparticles used in the previous researches was almost limited to spherical NCs with few exceptions for metal nanocubes, we need to develop innovative processing techniques that allow the electrostatic assembly of nanocubes into close-packed nanostructures. The author cannot disclose at present any concrete ideas for the realization of such techniques, but some experimental results concerning the fabrication of nanoparticulate layers and films on electrically patterned substrates may give a hint for solving the problems concerned $[37,38]$.

\section{Nanocrystal ceramic devices with novel electronic functions}

Figure 7 shows one example of $\mathrm{NC}$ ceramic devices with novel electronic functions, in which a schematic of a NC device made up of two kinds of ferroelectric (or dielectric) perovskite nanocubes assembled to form a specific polar domain structure, so-called polar nanoregion (PNR) inside which local spontaneous polarization exists, along with relaxor-type dielectric properties expected for the NC device. In Fig. 7 is also shown relaxor-like dielectric properties obtained for a free-standing dense ceramic sheet consisting of $\mathrm{BaTiO}_{3}$ NCs with a median size of $57 \mathrm{~nm} \mathrm{[39].} \mathrm{This}$ experimental fact provides a promising clue in considering the $\mathrm{NC}$ structures to be constructed for realizing $\mathrm{NC}$ devices with novel, gigantic dielectric functions based on relaxor-type dielectric responses. In the figure, $T_{\mathrm{d}}$ indicates so-called Burns temperature [40], below which PNRs exist; the free-standing $\mathrm{BaTiO}_{3} \mathrm{NC}$ ceramic sheet was confirmed to exhibit a definite (but slim) ferroelectric hysteresis loop at temperatures above the Curie point $T_{\mathrm{c}}$ up to around $150{ }^{\circ} \mathrm{C}$. Since intensive researches on relaxor ferroelectrics by Cross group [41] following the pioneering work of Smolenskii, there have been made a large number of investigations on the relaxor characteristics of complex perovskite materials, including $\mathrm{Pb}\left(\mathrm{Mg}_{1 / 3} \mathrm{Nb}_{2 / 3}\right) \mathrm{O}_{3}(\mathrm{PMN})$ as a representative one, with enormous interest from theoretical and engineering points of view. Since the relaxor dielectric behavior, characterized by a broad peak of dielectric constant with its large frequency dependence, has been observed in a variety of complex perovskite ferroelectrics, various models of the mechanism, including a diffuse phase transition (DPT) and spin-glass models, based on compositionally disordered structures have been proposed. However, recent theoretical and experimental (using X-ray and neutron analyses) studies have revealed that the origin

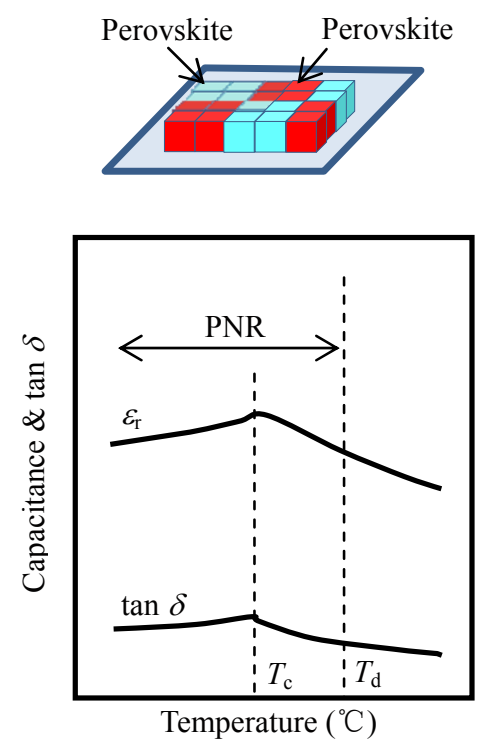

(A)

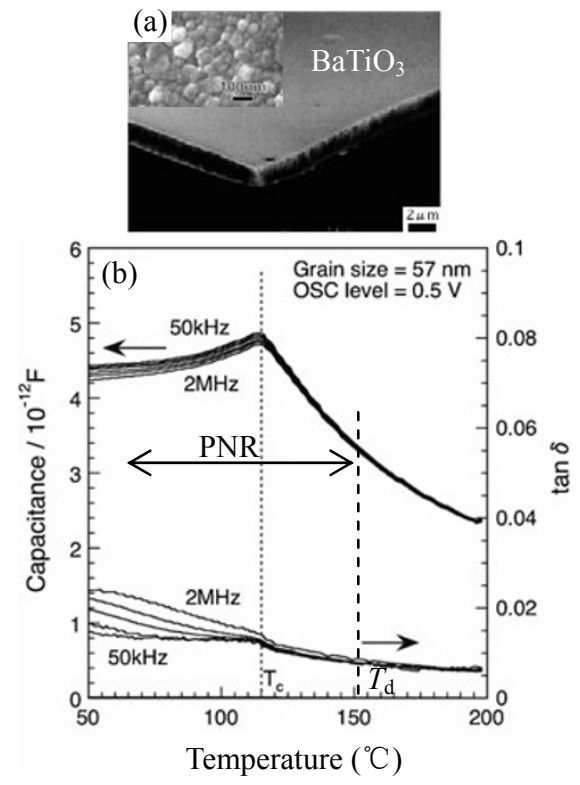

(B)

Fig. 7 (A) Schematic of a NC device with a specific polar domain structure and relaxor-type dielectric responses expected for the device, and (B) (a) SEM image of a free-standing $\mathrm{BaTiO}_{3} \mathrm{NC}$ ceramic sheet and (b) its dielectric properties observed. 
of relaxor is closely connected with the existence of PNRs induced by factors other than the structural phase transition seen in normal ferroelectrics. Various probable factors, including chemical/lattice disorder, stress/strain, grain size, and electric-field, which can induce the formation of PNRs through local fluctuations of spontaneous polarization, have been proposed, being still a point of controversy. In connection with this, if we are able to create such $\mathrm{NC}$ structures, for example, using $\mathrm{BaTiO}_{3}$ and $\mathrm{SrTiO}_{3}$ nanocubes, as those involving an effective PNR-inducing factor, lead-free NC ceramic devices with relaxor-type gigantic dielectric functions will be realized.

Figure 8 shows a new type of PTCR ceramic device made of a semiconducting $\mathrm{BaTiO}_{3} \mathrm{NC}$ sheet, taken as an example of $\mathrm{NC}$ ceramic devices with gigantic, intriguing semiconducting functions, for the realization of low-resistance flexible PTCR ceramic sheets being expected for applications in various areas including battery, medical science, and aerospace, as resettable circuit protection devices. The PTCR characteristic has been well known to be a grain boundary property of semiconducting $\mathrm{BaTiO}_{3}$ ceramics, and the Heywang-Jonker model has widely been accepted as one explaining most sufficiently its mechanism. The model explains the low-resistance of the PTCR characteristic below $T_{\mathrm{c}}$ by the effect of spontaneous polarization and the anomalous increase of resistance above the temperature by the decrease in dielectric constant of the materials according to the Curie-Weiss law [42]. The present author et al., however, has found the fact that many single grain boundaries in thin ceramic bars consisting of semiconducting $\mathrm{BaTiO}_{3}$ (doped with La) grains sintered together in series exhibit saw-tooth type PTCR characteristics, characterized by an abrupt increase in resistivity at $T_{\mathrm{c}}$, followed by a monotonous decrease in it, as shown in Fig. 8 [43]. The saw-tooth type PTCR characteristic can be hardly explained by the decrease of dielectric constant obeying the $\mathrm{C}-\mathrm{W}$ law alone, and to well explain the fact the effect of strain- and electric filed-induced polarization still existing above $T_{\mathrm{c}}$ needs to be taken account [44]. Regarding to the proposed PTCR ceramic nanostructure shown in Fig. 8, establishing a procedure to generate spontaneous polarization at both surface regions of the $\mathrm{BaTiO}_{3}$ nanocube sheet sandwiched with thin flexible conducting polymer sheets acting as electrodes is essential in the technology of fabricating this device. Any clear solutions for this problem have not been found yet, but the experimental results reporting the induced ferroelectricity in stained $\mathrm{BaTiO}_{3}$ and $\mathrm{SrTiO}_{3}$ thin films may provide some hints to solve the problems [45-47].

The technology of fabricating such NC ceramic

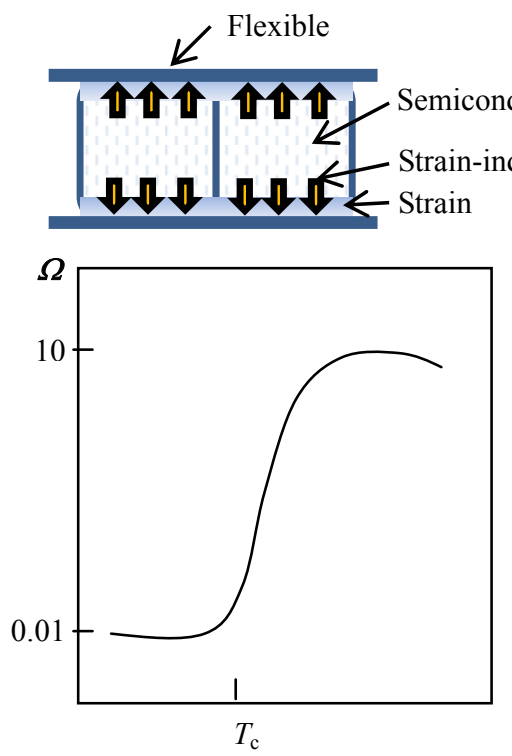

(A) (a)
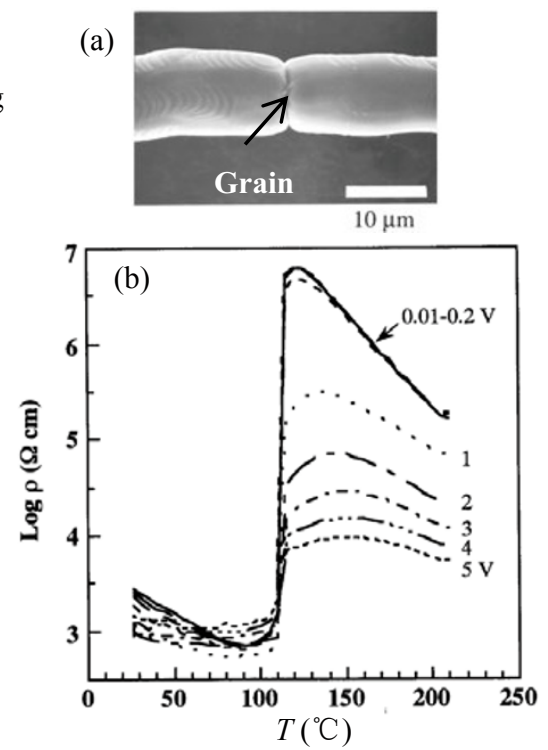

(B)

Fig. 8 (A) Schematic of a new type of PTCR device made of a semiconducting $\mathrm{BaTiO}_{3} \mathrm{NC}$ sheet and a low-resistance PTCR characteristic caused by strain-induced polarization generated in the NC sheet, and (B) (a) SEM image of a thin single-grain-boundary $\mathrm{BaTiO}_{3}$ ceramic bar and (b) single-grain-boundary PTCR characteristics observed. 
devices as are shown in Figs. 7 and 8 involves many problems which need to be solved; some of the essential problems are (a) synthesis of $\mathrm{BaTiO}_{3}$ and $\mathrm{SrTiO}_{3} \mathrm{NCs}$ with a regular cubic shape and narrow size distribution $(<10 \%$ standard deviation: SD), (b) crystallographic contact between nanocubes for providing them with good electronic interconnection, and (c) formation of novel electronic functional structures in NC ceramic devices. In regard to the problem (a), a number of investigations have been made of shape-control synthesis of metal NCs via solution-phase routes, and close understanding of the nucleation and growth mechanisms of a variety of metal NCs has been made possible. As a result, the technology of forming metal NCs with specific shapes has been established, which has allowed the synthesis of monodisperse nearly perfect cubic NCs of fcc (face-centered-cubic) metals, such as $\mathrm{Ag}, \mathrm{Au}$, and $\mathrm{Pd}$ [48]. However, the methodology established for the fcc metal systems is, basically, hardly extended to the systems of $\mathrm{BaTiO}_{3}$ and $\mathrm{SrTiO}_{3}$, so we need to establish procedures suitable for the systems. To this end, a deep insight into the forming mechanisms of $\mathrm{BaTiO}_{3}$ and $\mathrm{SrTiO}_{3}$ nanocubes synthesized in the previous studies $[21,22]$ should be effective. For the problem (b), making use of the OA phenomenon mentioned above seems to be effective to gain a most promising solution. To that end we need to establish a procedure for either replacing original surface ligands, particulary long-chain surfactant molecules such as oleic acid, with small molecules or removing them under proper $\mathrm{pH}$ conditions. The problem (c) is very difficult to find its solutions by current NC technology, but the author think that the introduction of local strains at specific sites of a nanostructure can be an effective solution and a challenging task in the $\mathrm{NC}$ technology of interest.

\section{Summary and outlook}

In this paper, a future prospect, with author's expectation for the next generation electronic ceramics, of the NC technology of fabricating ceramic devices with novel, gigantic electronic functions, which has attracted great interest in its potential for introducing a radical technical innovation into the current powder-based ceramic technology, is described. Since there have been many excellent reviews on the size/shape-controlled synthesis of colloidal NCs of a variety of materials and $\mathrm{NC}$ assembly technologies to construct electronic functional nanostructures, to avoid a mere reintroduction of such $\mathrm{NC}$ technologies and to make the point of the NC technology here concerned clear, the author here took up two NC ceramic devices of relaxor and PTCR, as representative examples relating to gigantic electronic functions, and $\mathrm{NC}$ technologies of the synthesis and assembly of perovskite oxide nanocubes, particularly $\mathrm{BaTiO}_{3}$ and $\mathrm{SrTiO}_{3}$ naocubes as building blocks in those cases. Since there have been only a few/few researches on the synthesis of perovskite oxide NCs with a regular cubic shape and a narrow size distribution of $<10 \% \mathrm{SD}$ and on the creation of nanostructures by assembly of oxide nanocubes, almost new, sophisticated $\mathrm{NC}$ technology needs to be established. The establishment of an innovative NC-based technology for ceramic devices with novel electronic functions is a challenging task in the field of electroceramics and is expected to be achieved in the near future.

\section{References}

[1] Pithan C, Hennings D, Waser R. Progress in the Synthesis of Nanocrystalline $\mathrm{BaTiO}_{3}$ Powders for MLCC. Int J Appl Ceram Technol 2005, 2: 1-14.

[2] Demirörs AF, Imhof A. $\mathrm{BaTiO}_{3}, \mathrm{SrTiO}_{3}, \mathrm{CaTiO}_{3}$, and $\mathrm{Ba}_{x} \mathrm{Sr}_{1-x} \mathrm{TiO}_{3}$ particles: A general approach for monodisperse colloidal perovskites. Chem Mater 2009, 21: 3002-3007.

[3] Dong W, Li B, Li Y, et al. General Approach to Well-Defined Perovskite $\mathrm{MTiO}_{3}(\mathrm{M}=\mathrm{Ba}, \mathrm{Sr}, \mathrm{Ca}$, and $\mathrm{Mg}$ ) Nanostructures. $J$ Phys Chem 2011, 115: 3918-3925.

[4] Yan T, Liu XL, Wang NR, et al. Synthesis of monodispersed barium titanate nanocrytals-hydrothermal recrystallization of $\mathrm{BaTiO}_{3}$ nanospheres. J Crystal Growth 2005, 281: 669-677.

[5] Polotai AV, Fujii I, Shay DP, et al. Effect of heating rates during sintering on the electrical properties of ultra-thin $\mathrm{Ni}-\mathrm{BaTiO}_{3}$ multilayer ceramic capacitors. $J$ Am Ceram Soc 2008, 91: 2540-2544.

[6] Niimi H, Mihara K, Sakabe Y, et al. Preparation of multilayer semiconducting $\mathrm{BaTiO}_{3}$ ceramics co-fired with Ni inner electrodes. Jpn J Appl Phys 2007, 46: 6715-6718.

[7] Wan S, Lu W, Wang X. Low-temperature sintering and electrical properties of $\mathrm{ZnO}-\mathrm{Bi}_{2} \mathrm{O}_{3}-\mathrm{TiO}_{2}-\mathrm{Co}_{2} \mathrm{O}_{3}-\mathrm{MnCO}_{3}$-Based varistor with $\mathrm{Bi}_{2} \mathrm{O}_{3}-\mathrm{B}_{2} \mathrm{O}_{3}$ frit for multilayer chip varistor applications. J Am Ceram Soc 2010, 93: 3319-3323. 
[8] Krishnaveni T, Kanth BR, Raju VSR, et al. Fabrication of multilayer chip inductors using $\mathrm{Ni}-\mathrm{Cu}-\mathrm{Zn}$ ferrites. $J$ Alloy Comp 2006, 414: 282-286.

[9] Shevchenko EV, Talapin DV, Kotov NA, et al. Structural diversity in binary nanoparticle superlattices. Nature 2006, 439: 55-59.

[10] Overgaag K, Evers W, Nijs B, et al. Binary superlattices of $\mathrm{PbSe}$ and $\mathrm{CdSe}$ nanocrystals. $\mathrm{J} \mathrm{Am}$ Chem Soc 2008, 130: 7833-7835.

[11] Talapin DV, Lee JS, Kovalenko MV, et al. Prospects of colloidal nanocrystals for electronic and optoelectronic applications. Chem Rev 2010, 110: 389-458.

[12] Luther JM, Law M, Beard MC, et al. Schottky solar cells based on colloidal nanocrystal films. Nano Lett 2008, 8: 3488-3492.

[13] Xia Y, Xiong Y, Lim B, et al. Shape-controlled synthesis of metal nanocrystals: Simple chemistry meets complex physics? Angew Chem Int Ed 2009, 48: $60-103$.

[14] Park J, Joo J, Kwon SG, et al. Synthesis of monodisperse spherical nanocrystals. Angew Chem Int Ed 2007, 46: 4630-4660.

[15] Rogach AL, Talapin DV, Shevchenko EV, et al. Organization of matter on different size scales: Monodisperse nanocrystals and their superstructures. Adv Funct Mater 2002, 12: 653-664.

[16] Jeong U, Teng X, Wang Y, et al. Superparamagnetic colloids: Controlled synthesis and niche applications. Adv Mater 2007, 19: 33-60.

[17] Clark IJ, Takeuchi T, Ohtoric N, et al. Hydrothermal synthesis and characterisation of $\mathrm{BaTiO}_{3}$ fine powders: Precursors, polymorphism and properties. $J$ Mater Chem 1999, 9: 83-91.

[18] Murray CB, Sun S, Doyle H, et al. Monodisperse 3d transition-metal $(\mathrm{Co}, \mathrm{Ni}, \mathrm{Fe})$ nanoparticles and their assembly into nanoparticle superlattices. MRS Bull 2001, 26: 985-991.

[19] Chandler CD, Roger C, Hampden-Smith MJ. Chemical aspects of solution routes to perovskite-phase mixed-metal. Chem Rev 1993, 93: 1205-1241.

[20] Wang X, Zhuang J, Peng Q, et al. A general strategy for nanocrystal synthesis. Nature 2005, 437: 121-124.

[21] Adireddy S, Lin C, Cao B, et al. Solution-based growth of monodisperse cubic-like $\mathrm{BaTiO}_{3}$ colloidal nanocrystals. Chem Mater 2010, 22: 1946-1948.

[22] Dang F, Mimura K, Kato K, et al. Growth of monodispersed $\mathrm{SrTiO} 3$ nanocubes by thermohydrolysis method. CrstEngComm 2011, 13:
3878-3883.

[23] Fujinami K, Katagiri K, Kamiya J, et al. Sub-10 nm strontium titanate nanocubes highly dispersed in non-polar organic solvents. Nanoscale 2010, 2: 2080-2083.

[24] Shevchenko EV, Talapin DV, Murray CB, et al. Structural characterization of self-assembled multifunctional binary nanoparticle superlattices. $J$ Am Chem Soc 2006, 128: 3620-3637.

[25] Dong A, Chen J, Vora PM, et al. Binary nanocrystal superlattice membranes self-assembled at the liquid-air interface. Nature 2010, 466: 474-477.

[26] Luther JM, Law M, Song Q, et al. Structural, optical, and electrical properties of self-assembled films of $\mathrm{PbSe}$ nanocrystals treated with 1,2-Ethanedithiol. ACS Nano 2008, 2: 271-280.

[27] Cölfen H, Antonietti M. Mesocrystals: Inorganic superstructures made by highly parallel crystallization and controlled alignment. Angew Chem Int Ed 2005, 44: 5576-5591.

[28] Konstantatos G, Howard I, Fischer A, et al. Ultrasensitive solution-cast quantum dot photodetectors. Nature 2006, 422: 180-183.

[29] Luther JM, Law M, Beard MC, et al. Schottky solar cells based on colloidal nanocrystal films. Nano Lett 2008, 8: 3488-3492.

[30] Penn R, Banfield JF. Morphology development and crystal growth in nanocrystalline aggregates under hydrothermal conditions: Insights from titania. Geochim Cosmochim Acta 1999, 63: 1549-1557.

[31] Du N, Zhang $\mathrm{H}$, Chen $\mathrm{B}$, et al. Ligand-free self-assembly of ceria nanocrystals into nanorods by oriented attachment at low temperature. J Phys Chem C 2007, 111: 12677-12680.

[32] Pacholski C, Kornowski A, Weller H. Self-assembly of $\mathrm{ZnO}$ : From nanodots to nanorods. Angew Chem Int Ed 2002, 41: 1188-1191.

[33] Cölfen H, Mann S. Higher-order organization by mesoscale self-assembly and transformation of hybrid nanostructures. Angew Chem Int Ed 2003, 42: 2350-2365.

[34] Penn RL, Oskam G, Strathmann TJ, et al. Epitaxial assembly in aged colloids. J Phys Chem B 2001, 105: 2177-2182.

[35] Leunissen ME, Christova CG, Hynninen AP, et al. Ionic colloidal crystals of oppositely charged particles. Nature 2005, 437: 235-240.

[36] Shipway AN, Lahav M, Gabai R, et al. Investigations into the electrostatically induced aggregation of $\mathrm{Au}$ nanoparticles. Langmuir 2000, 16: 8789-8795.

[37] Tzeng SD, Lin KJ, Hu JC, et al. Templated self-assembly of colloidal nanoparticles controlled 
by electrostatic nanopatterning on a $\mathrm{Si}_{3} \mathrm{~N}_{4} / \mathrm{SiO}_{2} / \mathrm{Si}$ electret. Adv Mater 2006, 18: 1147-1151.

[38] Chen KM, Jiang X, Kimerling LC, et al. Selective self-organization of colloids on patterned polyelectrolyte templates. Langmuir 2000, 16: 7825-7834.

[39] Shimooka H, Kohiki S, Kuwabara M. Characterization of barium titanate nanoparticles and dense nanograin free-standing films via sol-gel method using highly concentrated alkoxide solution. J Ceram Soc Jpn 2010, 118: 674-678.

[40] Burns G, Dacol FH. Glassy polarization behavior in ferroelectric compounds $\mathrm{Pb}\left(\mathrm{Mg}_{1 / 3} \mathrm{Nb}_{2 / 3}\right) \mathrm{O}_{3}$ and $\mathrm{Pb}\left(\mathrm{Zn}_{1 / 3} \mathrm{Nb}_{2 / 3}\right) \mathrm{O}_{3}$. Solid State Comm 1983, 48: 853-856.

[41] Viehland D, Jang SJ, Cross LE. Local polar configurations in lead magnesium niobate relaxors. $J$ Appl Phys 1991, 69: 414-419.

[42] Heywang W. Resistivity anomaly in doped barium titanate. J Am Ceram Soc 1964, 47: 484-490.

[43] Kuwabara M, Matsuda H, Hamamoto K. Giant piezoresistive effects in single grain boundaries of semi conducting barium titanate ceramics. $J$ Electroceram 1999, 4: 99-103.

[44] Hamamoto K, Kuwabara M. Effect of electric-field-induced polarization on positive temperature coefficient of resistivity characterisitics of semiconducting barium titanate ceramics. Jpn $J$ Appl Phys 2001, 40: L1163-L1165.

[45] Choi KJ, Biegalski M, Li YL, et al. Enhancement of ferroelectricity in strained $\mathrm{BaTiO}_{3}$ thin films. Science 2004, 306: 1005-1009.

[46] Harrington SA, Zhai J, Denev S, et al. Thick lead-free ferroelectric films with high Curie temperatures through nanocomposite induced strain. Nature Nanotech 2011, 6: 491-495.

[47] Haeni JH, Irvin P, Chang W, et al. Room-temperature ferroelectricity in strained $\mathrm{SrTiO}_{3}$. Nature 2004, 430: 758-761.

[48] Xiong Y, Xia Y. Shape-controlled synthesis of metal nanostructures: The case of palladium. Adv Mater 2007, 19: 3385-3391. 